\title{
HLA-DRB1 alleles in juvenile-onset systemic lupus erythematosus: renal histologic class correlations
}

B.L. Liphaus ${ }^{1}$, M.H.B. Kiss ${ }^{2}$ and A.C. Goldberg ${ }^{3}$

\author{
${ }^{1}$ Unidade de Reumatologia, Instituto da Criança, ${ }^{2}$ Faculdade de Medicina, \\ ${ }^{3}$ Laboratório de DNA e Imunologia, Instituto de Ciências Biomédicas, \\ Universidade de São Paulo, São Paulo, SP, Brasil
}

\begin{abstract}
Correspondence
B.L. Liphaus

Rua Teodoro Sampaio, 352/166

05406-000 São Paulo, SP

Brasil

Fax: +55-11-3898-1078

E-mail: bernadll@icr.hcnet.usp.br

Publication supported by FAPESP.

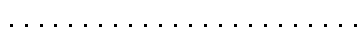

Received August 13, 2006

Accepted January 22, 2007

\section{Abstract}

Human leukocyte antigens (HLA) DRB1*03 and DRB1*02 have been associated with systemic lupus erythematosus (SLE) in Caucasians and black populations. It has been observed that certain HLA alleles show stronger associations with SLE autoantibodies and clinical subsets, although they have rarely been associated with lupus renal histologic class. In the present study, HLA-DRB1 allele correlations with clinical features, autoantibodies and renal histologic class were analyzed in a cohort of racially mixed Brazilian patients with juvenileonset SLE. HLA-DRB1 typing was carried out by polymerase chain reaction amplification with sequence-specific primers using genomic DNA from 55 children and adolescents fulfilling at least four of the American College of Rheumatology criteria for SLE. Significance was determined by the chi-square test applied to $2 \times 2$ tables. The HLA-DRB $1 * 15$ allele was most frequent in patients with renal, musculoskeletal, cutaneous, hematologic, cardiac, and neuropsychiatric involvement, as well as in patients positive for anti-dsDNA, anti-Sm, anti-U1-RNP, and anti-SSA/Ro antibodies, although an association between HLA alleles and SLE clinical features and autoantibodies could not be observed. The HLA-DRB $1 * 17$, HLA-DRB1*10, HLADRB1*15, and HLA-DRB1*07 alleles were significantly higher in patients with renal histologic class I, class IIA, class IIB, and class V, respectively. The present results suggest that the contribution of HLADRB1 alleles to juvenile-onset SLE could not be related to clinical or serological subsets of the disease, but it may be related to renal histologic classes, especially class I, class II A, class II B, and class V. The latter correlations have not been observed in literature.

\section{Introduction}

Systemic lupus erythematosus (SLE) is an autoimmune disease characterized by multisystem organ involvement and autoantibodies production (1). Genetic factors are likely to be important both in determining the overall susceptibility to SLE and influ-
Key words

- Systemic lupus erythematosus

- Human leukocyte antigen

- Lupus nephritis

- Juvenile-onset lupus

- HLA-DRB1 alleles encing the remarkable clinical heterogeneity of disease presentation and autoantibody production observed in affected subjects (2). Human leukocyte antigen (HLA) DRB $1 * 02$ and the allelic variant DRB1*15 have been associated with SLE in blacks and Asians and HLA-DRB $1 * 03$ has been associated with lupus in Caucasians, but clear associations 
with HLA and SLE could not be demonstrated in racially mixed populations (2-5). It is recognized that certain HLA alleles show stronger association with autoantibody repertories and clinical subsets than with SLE itself. Some studies have observed an association with HLA alleles and cutaneous or cardiac involvement (6). In addition, most studies showed an association between HLADRB $1 * 02$ and DRB $1 * 15$ and lupus nephritis, although HLA alleles have rarely been associated with SLE renal histologic class $(3,4,6,7)$. The association with HLA and SLE in children appears to be similar to that observed in adults, but clinical involvement, autoantibodies and renal histologic class frequencies are quite different in these two groups (8).

We conducted this study to determine HLA-DRB1 allele associations with SLE clinical subsets, autoantibodies and renal histologic class in a cohort of Brazilian children and adolescents.

\section{Patients and Methods}

\section{Patients}

We studied 55 consecutive Brazilian children and adolescents with SLE attending the Rheumatology Unit, Children's Institute, Faculty of Medicine, University of São Paulo. All patients met the American College of Rheumatology classification criteria for SLE (9). The study was approved by the local Ethics Committee and all patients or persons responsible gave written informed consent to participate. Patient mean age at disease onset was 10.4 years (range, 1-17 years), mean follow-up period was 3.5 years (range, 1-13 years) and 46 patients (84\%) were females (female:male ratio of 5.1:1). Clinical and serologic characteristics were recorded on a specific protocol form. The definitions of the clinical features were based on the American College of Rheumatology criteria (9). Forty-three patients underwent a renal biopsy and renal histologic class was established according to the World Health Organization (WHO) classification of lupus nephritis $(1,10)$.

\section{Laboratory investigations}

Serologic tests were performed in the same laboratory for all patients. Antinuclear antibodies were determined by indirect immunofluorescence using rat liver sections or human cell line HEp-2 as substrate (sera diluted 1/40). Anti-dsDNA antibodies were determined by indirect immunofluorescence with Crithidia luciliae as substrate (sera diluted 1/10). Precipitating antibodies to extractable nuclear antigens, including SSA/ Ro, SSB/La, U1-RNP and Sm were detected by enzyme-linked immunosorbent assay. The anti-ribosomal $\mathrm{P}$ antibody was also detected by enzyme-linked immunosorbent assay. A total of 14 HLA-DRB1 alleles $(* 01, * 15$, $* 16, * 17, * 18, * 04, * 11, * 12, * 13, * 14, * 07$, $* 08, * 09, * 10)$ were identified by polymerase chain reaction amplification with sequence-specific primers. The method was based on the procedure developed by Olerup and Zetterquist (11). Genomic DNA was extracted from peripheral blood mononuclear cells by the DTAB/CTAB method (12). Briefly, the buffy coat from $5 \mathrm{~mL}$ blood was vigorously mixed with $12 \%$ DTAB solution (12\% DTAB, $2.25 \mathrm{M} \mathrm{NaCl}, 150 \mathrm{mM}$ Tris, $\mathrm{pH}$ 8.6, 75 mM EDTA) and incubated for 5 min at $68^{\circ} \mathrm{C}$. After the addition of 2 volumes of chloroform, samples were vigorously mixed and centrifuged for $2 \mathrm{~min}$ at $10,000 \mathrm{~g}$ for separation into three phases. The upper phase was transferred to a fresh tube containing 2 volumes of $0.5 \%$ CTAB $(0.5 \%$ CTAB, $0.04 \mathrm{M} \mathrm{NaCl}$ ) and DNA was precipitated and further washed with $99.5 \%$ ethanol. PCR was carried out for 35 cycles under the following conditions: $200 \mathrm{ng}$ DNA, 10 $\mathrm{mM}$ Tris- $\mathrm{HCl}, \mathrm{pH} 8.4,50 \mathrm{mM} \mathrm{KCl}, 2 \mathrm{mM}$ $\mathrm{MgCl}_{2}, 0.001 \%$ gelatin, $0.2 \mathrm{mM}$ dNTP, 20 pmol of each primer, $2 \mathrm{U}$ Taq DNA poly- 
merase, and $\mathrm{H}_{2} \mathrm{O}$ up to $55 \mu \mathrm{L}$ were denatured for $1 \mathrm{~min}$ at $94^{\circ} \mathrm{C}$, annealed for $1 \mathrm{~min}$ at $55^{\circ} \mathrm{C}$, with an extension at $72^{\circ} \mathrm{C}$ for $1 \mathrm{~min}$. The PCR products were identified by $1.2 \%$ agarose gel electrophoresis.

\section{Statistical analysis}

To compare the HLA allele frequencies of SLE patients with and without the various clinical characteristics, autoantibodies and renal histologic class, the conventional chisquare test with Yates' correction for continuity or, when appropriate, the Fisher exact test (two-tailed) were used. The odds ratio was calculated with $2 \times 2$ contingency tables. The $95 \%$ confidence intervals were obtained using Cornfield's approximation. The P value was corrected for the number of alleles tested. The level of significance was set at $95 \%$ (13).

\section{Results}

The following clinical manifestations and autoantibodies were detected in this group of 55 children and adolescents with SLE: cutaneous involvement $(\mathrm{N}=53,96 \%)$, musculoskeletal involvement $(\mathrm{N}=42,76 \%)$, lung involvement $(\mathrm{N}=13,24 \%)$, cardiac involvement $(\mathrm{N}=23,42 \%)$, and neuropsychiatric involvement $(\mathrm{N}=25,45 \%)$; renal $(\mathrm{N}=45,82 \%)$ and hematologic $(\mathrm{N}=47$, $85 \%)$ abnormalities; 55 (100\%) had antinuclear antibodies, 38 (69\%) anti-dsDNA, 17 (31\%) anti-Sm, 20 (36\%) anti-U1-RNP, 19 (34\%) anti-SSA/Ro, and 6 (11\%) anti$\mathrm{SSB} / \mathrm{La}$ antibodies. Eleven patients were tested for the anti-ribosomal $\mathrm{P}$ antibody, which was positive in $3(27 \%)$.

HLA-DRB $1 * 15$, an allelic variant of $\mathrm{DRB} 1 * 02$, was more frequently detected in SLE patients with renal, musculoskeletal, cutaneous, hematologic, cardiac, and neuropsychiatric features compared with SLE patients without these clinical features, although a statistical significant association between
HLA alleles and SLE clinical involvement could not be demonstrated (Table 1). As expected, the HLA-DRB $1 * 15$ allele was more frequent in patients with positive antidsDNA, anti-Sm, anti-U1-RNP, and antiSSA/Ro antibodies; HLA-DRB1*04 and HLA-DRB ${ }^{*} 07$ were most frequent in patients with positive anti-SSB/La antibody; the HLA-DRB $1 * 13$ allele was more frequent in patients with positive anti-ribosomal $\mathrm{P}$ antibody, although the differences were not statistically significant (Table 2).

The patterns of glomerular damage seen in 43 renal biopsies according to the WHO classification of lupus nephritis were: minimal mesangial glomerulonephritis in 6 patients (14\%) (class I), mesangial glomerulonephritis A in 6 (14\%) (class IIA), mesangial

Table 1. Relative HLA phenotype frequency according to clinical manifestations in 55 children and adolescents with systemic lupus erythematosus.

\begin{tabular}{|c|c|c|c|c|c|}
\hline Clinical manifestations & HLA & $+(\%)$ & $-(\%)$ & OR & $95 \% \mathrm{Cl}$ \\
\hline Nephritis & DRB $1 * 15$ & $31.1 \%$ & $40.0 \%$ & 0.68 & $0.14-3.46$ \\
\hline Musculoskeletal involvement & DRB1*15 & $28.5 \%$ & $54.5 \%$ & 0.47 & $0.11-2.0$ \\
\hline Cutaneous involvement & DRB1*15 & $33.9 \%$ & $0.0 \%$ & - & - \\
\hline Hematologic abnormalities & DRB1*15 & $31.9 \%$ & $37.5 \%$ & 0.78 & $0.13-4.84$ \\
\hline Lung involvement & $\mathrm{DRB} 1{ }^{*} 04$ & $38.4 \%$ & $14.3 \%$ & 3.75 & $0.75-19.3$ \\
\hline Cardiac involvement & DRB1*15 & $30.4 \%$ & $34.4 \%$ & 0.84 & $0.23-3.04$ \\
\hline Cardiac involvement & $\mathrm{DRB} 1{ }^{*} 07$ & $30.4 \%$ & $21.8 \%$ & 1.56 & $0.39-6.26$ \\
\hline Neuropsychiatric involvement & DRB1*15 & $32.0 \%$ & $33.3 \%$ & 0.94 & $0.26-3.37$ \\
\hline Neuropsychiatric involvement & DRB1*13 & $32.0 \%$ & $56.7 \%$ & 2.35 & $0.56-10.2$ \\
\hline
\end{tabular}

$\mathrm{HLA}=$ human leukocyte antigen; $\mathrm{OR}=$ odds ratio; $95 \% \mathrm{Cl}=95 \%$ confidence interval. All $P$ values were $\geq 0.10$.

Table 2. Relative HLA phenotype frequency according to type of autoantibodies in 55 children and adolescents with systemic lupus erythematosus.

\begin{tabular}{lccccc}
\hline Autoantibodies & HLA & $+(\%)$ & $-(\%)$ & OR & $95 \% \mathrm{Cl}$ \\
\hline Anti-dsDNA & DRB1*15 & $31.5 \%$ & $22.2 \%$ & 0.85 & $0.22-3.34$ \\
Anti-Sm & DRB1 $^{*} 15$ & $47.1 \%$ & $26.3 \%$ & 2.49 & $0.65-9.77$ \\
Anti-U1-RNP & DRB1 $^{*} 15$ & $45.0 \%$ & $25.7 \%$ & 2.36 & $0.64-8.91$ \\
Anti-SSA/Ro & DRB1 $^{*} 15$ & $36.8 \%$ & $30.5 \%$ & 1.33 & $0.35-4.99$ \\
Anti-SSB/La & DRB1 $^{*} 04$ & $33.3 \%$ & $18.4 \%$ & 2.22 & $0.24-18.1$ \\
Anti-SSB/La & DRB1 $^{*} 07$ & $33.3 \%$ & $24.5 \%$ & 1.54 & $0.17-11.9$ \\
Anti-ribosomal P antibody & DRB1 ${ }^{*} 13$ & $66.6 \%$ & $21.2 \%$ & 8.40 & $0.51-262.3$
\end{tabular}

$\mathrm{HLA}=$ human leukocyte antigen; $\mathrm{OR}=$ odds ratio; $95 \% \mathrm{Cl}=95 \%$ confidence interval. All $P$ values were $\geq 0.13$. 
glomerulonephritis B in 8 (19\%) (class IIB), focal proliferative glomerulonephritis in 4 (9\%) (class III), diffuse proliferative glomerulonephritis in 4 (9\%) (class IV), and membranous glomerulonephritis in $15(35 \%)$ (class V).

HLA-DRB $1 * 17$, a subtype of DRB $1 * 03$, was positively associated with renal histologic class I $(\mathrm{P}=0.034)$ compared with SLE patients belonging to other renal histologic classes. Similarly, HLA-DRB $1 * 10$ was associated with class IIA $(\mathrm{P}=0.03)$, HLADRB1*15 with class IIB $(\mathrm{P}=0.011)$ and HLA-DRB $1 * 07$ with class $V(P=0.012)$, although the differences were not statistically significant after correction for the number of comparisons (Table 3).

\section{Discussion}

Multiple loci can contribute to SLE susceptibility and disease pathogenesis is expressed as a complex genetic interaction that is undoubtedly influenced by environmental factors (2). Genetic studies of SLE are difficult because the disease is heterogeneous and can be divided into several clinical and immunologic subsets, each with its own genetic background (2). Furthermore, genes predisposing to SLE may vary according to

Table 3. Relative HLA phenotype frequency according to renal histologic class in 55 children and adolescents with systemic lupus erythematosus.

\begin{tabular}{|c|c|c|c|c|c|c|}
\hline Renal histologic class & HLA & $+(\%)$ & $-(\%)$ & Pc value & OR & $95 \% \mathrm{Cl}$ \\
\hline Class I & $\mathrm{DRB}^{*} 17$ & $50.0 \%$ & $10.2 \%$ & 0.47 & 8.80 & $1.03-82.4$ \\
\hline \multirow[t]{4}{*}{ Class IIA } & $\mathrm{DRB} 1{ }^{*} 01$ & $33.3 \%$ & $16.3 \%$ & - & 2.56 & $0.27-21.46$ \\
\hline & DRB $1 * 11$ & $33.3 \%$ & $10.2 \%$ & - & 4.40 & $0.42-42.56$ \\
\hline & $\mathrm{DRB} 1{ }^{*} 07$ & $33.3 \%$ & $24.5 \%$ & - & 1.54 & $0.17-11.94$ \\
\hline & $\mathrm{DRB}^{*}{ }^{*} 10$ & $33.3 \%$ & $2.1 \%$ & 0.42 & 24.0 & $1.25-878.2$ \\
\hline Class IIB & DRB1*15 & $75.0 \%$ & $25.5 \%$ & 0.15 & 8.75 & $1.30-73.7$ \\
\hline \multirow[t]{2}{*}{ Class III } & $\mathrm{DRB}^{*}{ }^{*} 15$ & $50.0 \%$ & $31.5 \%$ & - & 2.19 & $0.20-24.6$ \\
\hline & DRB1*07 & $50.0 \%$ & $23.5 \%$ & - & 3.25 & $0.28-37.5$ \\
\hline \multirow[t]{2}{*}{ Class IV } & $\mathrm{DRB} 1 * 17$ & $50.0 \%$ & $11.7 \%$ & - & 7.50 & $0.60-98.9$ \\
\hline & $\mathrm{DRB} 1{ }^{*} 08$ & $50.0 \%$ & $13.7 \%$ & - & 6.29 & $0.51-79.6$ \\
\hline Class V & $\mathrm{DRB} 1{ }^{*} 07$ & $53.0 \%$ & $15.0 \%$ & 0.16 & 6.48 & $1.43-31.0$ \\
\hline
\end{tabular}

$\mathrm{HLA}=$ human leukocyte antigen; $\mathrm{OR}=$ odds ratio; $95 \% \mathrm{Cl}=95 \%$ confidence interval; $P c$ value $=P$ value corrected for 14 comparisons. patient ethnic group $(2,3)$.

Several studies, including association and family investigations, have confirmed the linkage of the SLE and MHC region in humans. The HLA-DRB $1 * 03$ allele has been associated with SLE in Caucasian populations while the HLA-DRB $1 * 02$ allele and the HLA-DRB $1 * 15$ and HLA-DRB $1 * 16$ subtypes have been associated with SLE in black and Asian ethnic groups (2-4,6,1421). The association of HLA-DRB $1 * 07$ and HLA-DRB $1 * 13$ with lupus was observed in a few studies, such as that by Wilson et al. (22) on African Americans and that by Cowland et al. (23) in Denmark. Barron et al. (24) showed similar associations in children with SLE: HLA-DR3 (DRB1*0301) in Caucasians and HLA-DR2 (DRB1*1503/ DRB1*1501), DRB5*0101, DQA1*0102, and DQB1*0602 in blacks. In Taiwanese patients with juvenile-onset SLE there was an association with HLA-DRB1*1602 (21). Silva and Donadi (25), studying 93 Brazilian SLE patients aged 13 to 66 years, observed a higher frequency of HLA-DRB $1 * 03$, a result that differs from that observed in our previous study on 55 children and adolescents with SLE in which HLA-DRB $1 * 15$ was the most frequent allele (5), and from that reported by Fernandes et al. (26) in a study on 56 adult patients with SLE in which HLA-DRB $1 * 02$ was the most frequent allele.

It has been suggested that certain MHC haplotypes show stronger association with SLE clinical manifestations and autoantibodies than with the disease itself $(3,4)$. In infants, SLE has been reported to be clinically and serologically different, with a higher frequency of renal involvement and mortality compared to adults $(1,8)$. These differences raise some questions regarding the possibility that childhood SLE could be a disease with its own genetic background.

We observed that the HLA-DRB1*15 allele was most frequent in patients with cutaneous, cardiac, neuropsychiatric, renal, 
and hematologic involvement, although an association could not be demonstrated. Doherty et al. (6) described an association with HLA-DR15 and SLE skin involvement in Chinese patients and Reivelle et al. (27) demonstrated an association with HLA-DR53 and HLA-DQA1 and malar erythema photosensitivity. Doherty et al. (6) also reported an association with the HLA-DR7 and HLADR9 alleles and cardiac involvement in Chinese patients. None of our patients with cardiac involvement presented the HLADRB1*09 allele. In the Brazilian study of Silva and Donadi (25), HLA-DR3, HLADR9 and HLA-DQ2 were the most frequent alleles in SLE patients with neuropsychiatric involvement, a result that differs from that observed in the present study. Fronek et al. (7) and Doherty et al. (6) described an association with HLA-DR2 and SLE nephritis in Americans. Lupus nephritis has also been associated with the HLA-DR3, HLADR15 and HLA-DR16 alleles in different ethnic groups $(6,7,28)$. There is no description in the literature of an association between HLA alleles and SLE hematologic involvement. Galeazzi et al. (28) described an association between HLA-DRB $1 * 03$ and lung involvement in SLE. Alternatively, HLA-DRB1*04 was the most frequent allele in our patients with lung abnormality.

Some associations have been described concerning the HLA-DR alleles and SLE autoantibodies. The anti-dsDNA antibody has been associated with the HLA-DR2, DR3, DR7, and DR15 alleles (2,28). The anti-Sm antibody has been associated with the HLADR2, HLA-DR4 and HLA-DR7 alleles, the anti-U1-RNP antibody with HLA-DR4, and the anti-SSA/Ro and anti-SSB/La antibodies with the HLA-DR2 and HLA-DR3 alleles $(3,4,28-32)$. The HLA-DRB1*15 allele was most frequent in our patients with positive anti-dsDNA, anti-Sm, anti-U1-RNP, and anti-SSA/Ro antibodies, although an association could not be observed. Additionally, the anti-ribosomal $\mathrm{P}$ antibody has been found to be associated with the HLA-DR4 and DR2 (DRB1*1501 or DRB1*1503) alleles $(2,33)$. None of our patients positive for antiribosomal $\mathrm{P}$ presented the HLA-DR4 or HLA-DR2 allele. For the interpretation of the present study it should be considered that clear associations between HLA region genes and SLE clinical and serological features could not be demonstrated because genes within or in linkage with the HLA region, such as DQ, DP, TNF- $\alpha$, and C4 were not evaluated $(14,16,19,28,34)$.

Renal histologic class is an important prognostic factor for SLE patients. Nevertheless, HLA alleles have rarely been associated with SLE renal histologic class. The renal histologic class frequencies observed in the present study were similar to those reported in the literature $(1,8)$. Interestingly, 3 of 6 patients belonging to WHO renal histologic class I showed HLA-DRB1*17, an allelic variant of DRB $1 * 03$. HLA-DRB $1 * 03$ has been associated with SLE patients with disease onset after 35 years of age, who have less frequent and less severe renal involvement (2), possibly suggesting a protective effect of the HLA-DRB $1 * 03$ allele for lupus nephritis. On the other hand, Shankarkumar et al. (35), studying 53 patients with severe SLE who had one or more organ affected, such as kidney, brain, heart, and lungs, observed that the HLA-DRB1*03 allele was significantly increased in this group. Only 3 of the 55 children and adolescents with SLE presented HLA-DRB $1 * 10$ and 2 of them belonged to renal histologic class IIA. Eight patients belonged to class IIB and 6 of them presented the HLA-DRB $1 * 15$ allele, although our study could not demonstrate an association between HLA-DRB $1 * 15$ and lupus nephritis per se. Fifteen patients had membranous glomerulonephritis (class V) and 8 presented HLA-DRB $1 * 07$. These findings are different from those observed by Marchini et al. (36), who showed an association between HLA-DRB1*15 and severe form of lupus glomerulonephritis class III 
and class IV. Interestingly, Endreffy et al. (37) observed that the DRB $1 * 1501$ allele was less frequent in patients with lupus nephritis. In contrast, HLA-DRB $1 * 03$ and $\mathrm{DRB} 1 * 07$ alleles were more frequent in patients with lupus nephritis. In addition, HLADR expression was significantly increased in kidney arteries and arterioles of SLE patients compared to controls, although it did not correlate with WHO renal histologic class (38). The extended HLA-A1,B8,DR3 haplotype has been associated with idiopathic membranoproliferative glomerulonephritis and with SLE (39). The individual
HLA-DR3, HLA-DR15 and HLA-DR7 components have been associated with membranous idiopathic nephropathy, membranoproliferative glomerulonephritis and idiopathic nephrotic syndrome, respectively (39). Recently, a Brazilian study observed an association between DD genotype of the angiotensin-converting enzyme and SLE renal histologic classes III and IV (40).

The present results suggest that the contribution of MHC genes to SLE cannot be only related to the clinical and serological subsets of the disease but also to its renal histologic class.

\section{References}

1. Cassidy JT, Petty RE. Systemic lupus erythematosus. In: Cassidy JT, Petty RE (Editors), Textbook of pediatric rheumatology. 3rd edn. Philadelphia: WB Saunders; 1995. p 260-322.

2. Arnett FC. The genetics of human lupus. In: Wallace DJ, $\mathrm{Ham} \mathrm{BH}$ (Editors), Dubois' lupus erythematosus. 5th edn. Philadelphia: Williams \& Wilkins; 1997. p 77-117.

3. Schur PH. Genetics of systemic lupus erythematosus. Lupus 1995; 4: 425-437.

4. Arnett FC. Genetic aspects of human lupus. Clin Immunol Immunopathol 1992; 63: 4-6.

5. Liphaus BL, Goldberg AC, Kiss MH, Silva CA. Analysis of human leukocyte antigens class II-DR in Brazilian children and adolescents with systemic lupus erythematosus. Rev Hosp Clin Fac Med São Paulo 2002; 57: 277-282.

6. Doherty DG, Ireland R, Demaine AG, Wang F, Veerapan K, Welsh $\mathrm{KI}$, et al. Major histocompatibility complex genes and susceptibility to systemic lupus erythematosus in southern Chinese. Arthritis Rheum 1992; 35: 641-646.

7. Fronek Z, Timmerman LA, Alper CA, Hahn BH, Kalunian K, Peterlin $\mathrm{BM}$, et al. Major histocompatibility complex genes and susceptibility to systemic lupus erythematosus. Arthritis Rheum 1990; 33: 15421553.

8. Antolin J, Amerigo MJ, Cantabrana A, Roces A, Jimenez P. Systemic lupus erythematosus: clinical manifestations and immunological parameters in 194 patients. Subgroup classification of SLE. Clin Rheumatol 1995; 14: 678-685.

9. Hochberg MC. Updating the American College of Rheumatology revised criteria for the classification of systemic lupus erythematosus. Arthritis Rheum 1997; 40: 1725.

10. Cameron JS. Lupus nephritis in childhood and adolescence. Pediatr Nephrol 1994; 8: 230-249.

11. Olerup $\mathrm{O}$, Zetterquist $\mathrm{H}$. HLA-DR typing by PCR amplification with sequence-specific primers (PCR-SSP) in 2 hours: an alternative to serological DR typing in clinical practice including donor-recipient matching in cadaveric transplantation. Tissue Antigens 1992; 39: 225-235.
12. Gustincich S, Manfioletti G, Del Sal G, Schneider C, Carninci P. A fast method for high-quality genomic DNA extraction from whole human blood. Biotechniques 1991; 11: 298-302.

13. Fukuda K, Sugawa K, Wakisaka A, Moriuchi J, Matsuura N, Sato Y. Statistical detection of HLA and disease association. Tissue Antigens 1985; 26: 81-86.

14. Davies EJ, Steers G, Ollier WE, Grennan DM, Cooper RG, Hay EM, et al. Relative contributions of HLA-DQA and complement C4A loci in determining susceptibility to systemic lupus erythematosus. $\mathrm{Br} J$ Rheumatol 1995; 34: 221-225.

15. Hashimoto $H$, Nishimura $Y$, Dong RP, Kimura A, Sasazuki $T$, Yamanaka K, et al. HLA antigens in Japanese patients with systemic lupus erythematosus. Scand J Rheumatol 1994; 23: 191-196.

16. Hong GH, Kim HY, Takeuchi F, Nakano K, Yamada H, Matsuta K, et al. Association of complement C4 and HLA-DR alleles with systemic lupus erythematosus in Koreans. J Rheumatol 1994; 21: 442-447.

17. Batchelor JR. Systemic lupus erythematosus and genes within the HLA region. Br J Rheumatol 1993; 32: 13-15.

18. Goldstein R, Sengar DP. Comparative studies of the major histocompatibility complex in French Canadian and non-French Canadian Caucasians with systemic lupus erythematosus. Arthritis Rheum 1993; 36: 1121-1127.

19. Hartung K, Baur MP, Coldewey R, Fricke M, Kalden JR, Lakomek $\mathrm{HJ}$, et al. Major histocompatibility complex haplotypes and complement C4 alleles in systemic lupus erythematosus. Results of a multicenter study. J Clin Invest 1992; 90: 1346-1351.

20. Rudwaleit M, Gibson K, Wordsworth P, Pile K, Oh V. HLA associations of systemic lupus erythematosus in Chinese from Singapore. Ann Rheum Dis 1995; 54: 686-687.

21. Huang JL, Shaw CK, Lee A, Lee TD, Chou YH, Kuo ML. HLA-DRB1 antigens in Taiwanese patients with juvenile-onset systemic lupus erythematosus. Rheumatol Int 2001; 21: 103-105.

22. Wilson WA, Scopelitis E, Michalski JP. Association of HLA-DR7 with both antibody to $S S A(R o)$ and disease susceptibility in blacks with systemic lupus erythematosus. J Rheumatol 1984; 11: 653-657.

23. Cowland JB, Andersen V, Halberg P, Morling N. DNA polymorphism 
of HLA class II genes in systemic lupus erythematosus. Tissue Antigens 1994; 43: 34-37.

24. Barron KS, Silverman ED, Gonzales J, Reveille JD. Clinical, serologic, and immunogenetic studies in childhood-onset systemic lupus erythematosus. Arthritis Rheum 1993; 36: 348-354.

25. Silva LM, Donadi EA. Is immunogenetic susceptibility to neuropsychiatric systemic lupus erythematosus (SLE) different from nonneuropsychiatric SLE? Ann Rheum Dis 1996; 55: 544-547.

26. Fernandes SR, Persoli LB, Marques SB, Costallat LT. HLA antigens and susceptibility to systemic lupus erythematosus in Brazilian patients. Rev Bras Reumatol 1998; 38: 332-336.

27. Reveille JD, Anderson KL, Schrohenloher RE, Acton RT, Barger BO. Restriction fragment length polymorphism analysis of HLA-DR, $\mathrm{DQ}, \mathrm{DP}$ and C4 alleles in Caucasians with systemic lupus erythematosus. J Rheumatol 1991; 18: 14-18.

28. Galeazzi M, Sebastiani GD, Morozzi G, Carcassi C, Ferrara GB, Scorza R, et al. HLA class II DNA typing in a large series of European patients with systemic lupus erythematosus: correlations with clinical and autoantibody subsets. Medicine 2002; 81: 169-178.

29. Olsen ML, Arnett FC, Reveille JD. Contrasting molecular patterns of MHC class II alleles associated with the anti-Sm and anti-RNP precipitin autoantibodies in systemic lupus erythematosus. Arthritis Rheum 1993; 36: 94-104.

30. Galeazzi M, Sebastiani GD, Passiu G, Angelini G, Delfino L, Asherson RA, et al. HLA-DP genotyping in patients with systemic lupus erythematosus: correlations with autoantibody subsets. J Rheumatol 1992; 19: 42-46.

31. Julkunen $H$, Siren MK, Kaaja R, Kurki P, Friman C, Koskimies S. Maternal HLA antigens and antibodies to SS-A/Ro and SS-B/La. Comparison with systemic lupus erythematosus and primary Sjögren's syndrome. Br J Rheumatol 1995; 34: 901-907.

32. Hamilton RG, Harley JB, Bias WB, Roebber M, Reichlin M, Hochberg $\mathrm{MC}$, et al. Two Ro (SS-A) autoantibody responses in systemic lupus erythematosus. Correlation of HLA-DR/DQ specificities with quantitative expression of Ro (SS-A) autoantibody. Arthritis Rheum 1988; 31: 496-505.

33. Teh LS, Doherty DG, Williams BD. HLA-DRB genes and antiribosomal $\mathrm{P}$ antibodies in systemic lupus erythematosus. $\mathrm{Br} J$ Rheumatol 1994; 33: 1125-1126.

34. Jonsen A, Bengtsson AA, Sturfelt G, Truedsson L. Analysis of HLA DR, HLA DQ, C4A, FcgammaRIla, FcgammaRIIla, MBL, and IL-1Ra allelic variants in Caucasian systemic lupus erythematosus patients suggests an effect of the combined FcgammaRIla R/R and IL-1Ra 2/2 genotypes on disease susceptibility. Arthritis Res Ther 2004; 6: R557-R562.

35. Shankarkumar U, Ghosh K, Badakere SS, Mohanty D. HLADRB1*03 and DQB1*0302 associations in a subset of patients severely affected with systemic lupus erythematosus from western India. Ann Rheum Dis 2003; 62: 92-93.

36. Marchini M, Antonioli R, Lleo A, Barili M, Caronni M, Origgi L, et al. HLA class II antigens associated with lupus nephritis in Italian SLE patients. Hum Immunol 2003; 64: 462-468.

37. Endreffy E, Kovacs A, Kovacs L, Pokorny G. HLA class II allele polymorphism in Hungarian patients with systemic lupus erythematosus. Ann Rheum Dis 2003; 62: 1017-1018.

38. Cheah PL, Looi LM, Chua CT, Yap SF, Fleming S. Enhanced major histocompatibility complex (MHC) class II antigen expression in lupus nephritis. Malays J Pathol 1997; 19: 115-120.

39. Bishof NA, Welch TR, Beischel LS, Carson D, Donnelly PA. DP polymorphism in HLA-A1,-B8,-DR3 extended haplotypes associated with membranoproliferative glomerulonephritis and systemic lupus erythematosus. Pediatr Nephrol 1993; 7: 243-246.

40. Sprovieri SR, Sens YA, Martini FD. Association between polymorphisms of the renin-angiotensin system and more severe histological forms of lupus nephritis. Clin Nephrol 2005; 64: 20-27. 